\title{
New diagnosis of von Hippel-Lindau on CT
}

\author{
Brian Reed Curtis ${ }^{1} \cdot$ Eric E. Curtis ${ }^{2}$
}

Received: 1 October 2016/Accepted: 8 October 2016/Published online: 21 October 2016

(C) SIMI 2016

\section{Patient history and presentation}

A 38-year-old woman presented to the emergency department with weakness and ambulatory dysfunction. She had been experiencing weakness in all four extremities for some time, but she experienced a recent progression of ambulatory dysfunction, and this prompted her visit to the hospital. She was alert and oriented, but weak in all four extremities on physical exam. She had no significant past medical or surgical history. Her father and two of her sisters have von Hippel-Lindau disease. Laboratory studies, including complete blood count, basic metabolic panel, and liver function tests, including a coagulation panel, were within normal limits. Microscopic hematuria was identified on urinalysis. A computed tomography (CT scan) of the chest, abdomen, and pelvis was ordered for further evaluation.

\section{Imaging findings}

The lung parenchyma, mediastinum, and chest wall were normal. Evaluation of the abdomen showed a normal liver and spleen. The first abnormalities identified were multiple pancreatic cystic lesions, one of which was a

Brian Reed Curtis

brian.curtis@jefferson.edu

1 Thomas Jefferson University Hospitals, Philadelphia, PA, USA

2 Virginia Commonwealth University School of Medicine, Richmond, VA, USA multi-lobulated cystic lesion in the pancreatic head, characteristic of a serous cystadenoma (Fig. 1). The adrenal glands were normal, but in the lower pole of the left kidney, there was an enhancing solid mass (Fig. 2). The remainder of the pelvis was normal. After evaluating the abdominopelvic organs, the spine was evaluated, and multiple hyper-enhancing lesions within the spinal canal were identified. They were predominantly located eccentrically along the dorsal spinal canal, and were well-defined. The largest were identified at levels T8, T11, and L1-L2 (Fig. 3). The constellation of findings was consistent with von Hippel-Lindau disease with pancreatic cystic lesions, including a serous cystadenoma in the pancreatic head, a left lower pole renal cell carcinoma, which explained her microscopic hematuria, and multiple spinal hemangioblastomas, which contributed to her presenting symptom of weakness. Magnetic resonance imaging (MRI) of the neural axis was performed, and confirmed multiple enhancing posterior fossa and spinal hemangioblastomas.

\section{Clinical course}

The patient underwent urgent suboccipital craniotomy with cerebellar, medullary, and cervical intramedullary spinal tumor resection with $\mathrm{C} 1$ to $\mathrm{C} 3$ laminectomies and laminoplasties due to the presence of obstructive hydrocephalus and weakness. Pathology examination confirmed cerebellar, brainstem, and cervical spinal cord hemangioblastomas, WHO grade I. She still has weakness in all extremities, but it is showing some improvement with therapy. She is being followed by urology regarding her renal mass, and continues to follow with neurosurgery. 
Fig. 1 Axial (a) and coronal (b) CT images of the abdomen show pancreatic cysts (arrows in b), including a well-defined multi-lobulated radially oriented lesion in the pancreatic head characteristic of a serous cystadenoma (arrow in a)
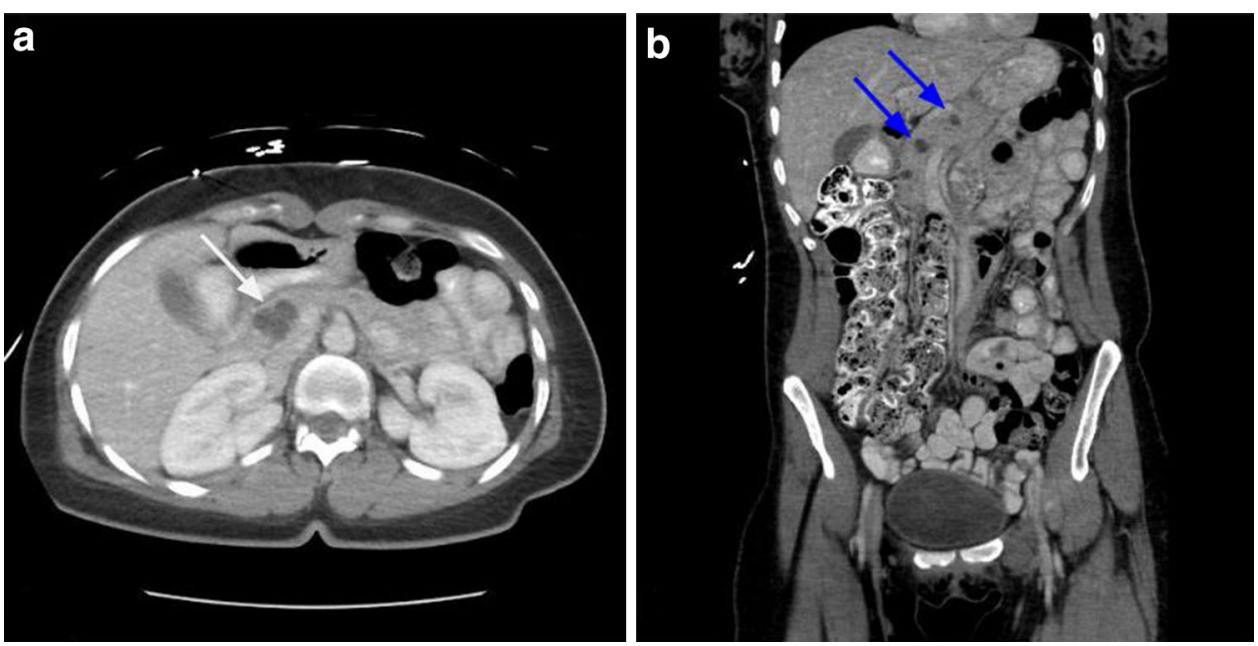

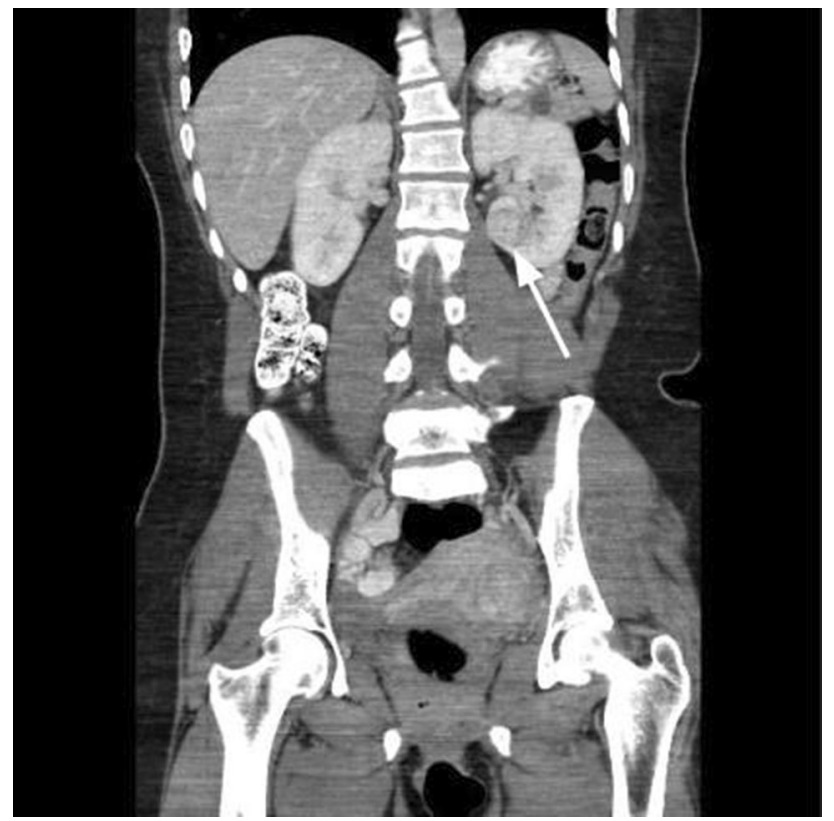

Fig. 2 Coronal CT of the abdomen shows an enhancing renal cell carcinoma in the left lower kidney (arrow)

\section{Discussion}

Von Hippel-Lindau disease (vHL) is an autosomal dominantly inherited multi-system disorder related to the inactivation of a tumor suppressor gene on chromosome 3 p25
[1]. Patients with vHL have a variety of benign and malignant tumors, including retinal and central nervous system hemangioblastomas, renal cysts and malignancies, pancreatic cysts and tumors, pheochromocytoma, and endolymphatic sac tumors [2]. Our patient has cerebellar hemangioblastomas (identified in 44-72\% of vHL patients), a medullary hemangioblastoma (identified in $5 \%$ of vHL patients), spinal cord hemangioblastomas (identified in $13-59 \%$ of vHL patients), pancreatic cysts (identified in $50-91 \%$ of vHL patients), including a serous cystadenoma (identified in $12 \%$ of vHL patients), and a renal cell carcinoma (identified in $24-45 \%$ of vHL patients). Pancreatic cysts and enhancing renal masses are readily identifiable on abdominal $\mathrm{CT}$ scan; however, the spinal canal is a common blind spot on CT scan and requires dedicated attention [3]. Even when the CT scan is performed to evaluate the abdominopelvic viscera or the lung parenchyma and mediastinum, the spinal canal requires devoted consideration. Our patient had pancreatic cysts and a renal cell carcinoma leading the interpreting physician to closely inspect the spinal canal, since VHL was the likely diagnosis; however, patients with vHL can present with only CNS hemangioblastomas and no visceral involvement [4]. The initial diagnosis of vHL was made on CT scan with the constellation of pancreatic cysts, renal cell carcinoma, and spinal hemagioblastomas, which explained the patient's presenting symptoms of weakness, 
Fig. 3 Axial (a) and sagittal (b) CT images show enhancing thoracic and lumbar spinal canal hemangioblastomas (arrows)
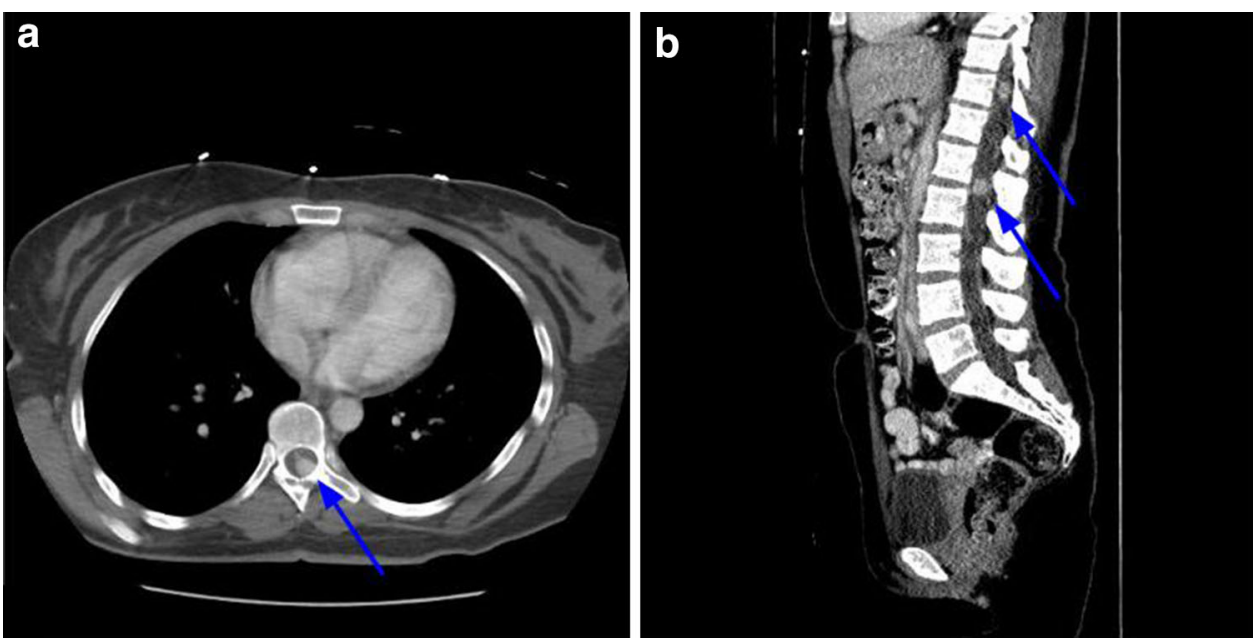

and could have been missed if specific attention was not paid to the spinal canal.

\section{Compliance with ethical standards}

Conflict of interest The authors declare that they have no conflict of interest.

Statement of human and animal rights This article does not contain any studies with human participants or animals performed by any of the authors.

Informed consent For this type of study formal consent is not required.

\section{References}

1. Seizinger BR, Rouleau GA, Ozelius LJ et al (1988) Von Hippel Lindau disease maps to the region of chromosome 3 associated with renal cell carcinoma. Nature 332(6161):268-269

2. Leung Rebecca S, Biswas Sona V, Duncan Mark, Rankin Sheila (2008) Imaging features of Von Hippel-Lindau disease. RadioGraphics 28(1):65-79

3. Wu Carol C, Khorashadi Leila, Abbott Gerald F, Shepard Jo-Anne O (2013) Common blind spots on chest CT: where are they all hiding? Part 2, Extrapulmonary structures. Am J Roentgenol 201:5

4. Melmon KL, Rosen SW (1964) Von Hippel Lindau's disease: review of the literature and study of a large kindred. Am J Med 36:595-617 